Available online at www.eccomasproceedia.org

Eccomas Proceedia COMPDYN (2019) 673-689

ECCOMAS

Proceedia
COMPDYN 2019

$7^{\text {th }}$ ECCOMAS Thematic Conference on Computational Methods in Structural Dynamics and Earthquake Engineering M. Papadrakakis, M. Fragiadakis (eds.) Crete, Greece, 24-26 June 2019

\title{
STOCHASTIC ASSESSMENT OF ROCKING MASONRY FAÇADES UNDER REAL SEISMIC RECORDS
}

\author{
*Linda Giresini ${ }^{1}$, Francesca Taddei ${ }^{2}$, Claudia Casapulla ${ }^{3}$, Gerhard Müller ${ }^{2}$ \\ ${ }^{1}$ Department of Energy, Systems, Territory and Constructions Engineering, University of Pisa, Largo Lucio \\ Lazzarino, 1, 56100, Pisa, Italy; linda.giresini@unipi.it. \\ ${ }^{1}$ Lehrstuhl für Baumechanik, Technische Universität München Arcisstr. 21, 80333 München; francesca.tad- \\ dei@tum.de, gerhard.mueller@tum.de \\ ${ }^{3}$ Dipartimento di Strutture per l'Ingegneria e l'Architettura, Università di Napoli Federico II, Via Forno Vecchio \\ 36, 80134, Napoli, Italy; casacla@unina.it \\ *Presenting author
}

\begin{abstract}
This contribution assesses in stochastic terms the safety level of masonry façades potentially subjected to out-of-plane loading and the beneficial effect offered by horizontal restraints such as steel tie rods - under earthquakes. A parametric analysis is performed on façades of different slenderness and size to state their influence on the response in probabilistic terms, showing that velocity and energy based Intensity Measures are optimal since they usually respect the efficiency and practicality criteria. In case of retrofitting interventions, the univariate FCs can be plotted in the two configurations (with and without steel tie rods). In order to effectively present the results, the graph of the difference of conditional probability is elaborated, which directly gives the information on the earthquake intensity for which the level of improvement is more relevant. Univariate and bivariate curves are also compared and the former may be not in favor of safety, especially for stronger seismic records.

This probabilistic procedure is ideal for applications in earthquake engineering, assessing in stochastic sense the level of improvement obtained with traditional retrofitting solutions.
\end{abstract}

Keywords: rocking, fragility curves, horizontal restraints, tie-rods, existing masonry, historic masonry

ISSN:2623-3347 @ 2019 The Authors. Published by Eccomas Proceedia.

Peer-review under responsibility of the organizing committee of COMPDYN 2019.

doi: $10.7712 / 120119.6948 .19345$ 


\section{INTRODUCTION}

The out-of-plane (OOP) behavior of masonry buildings is a crucial issue in the seismic vulnerability assessment of historic structures. Indeed, usually the connections between vertical elements and horizontal diaphragms and vertical walls are poor and the box-type behavior is not guaranteed. Also building irregularities could activate torsional modes that might cause a concentration of seismic demand in specific parts of the building [1,2]. The traditional tie-rods, generally used for improving the OOP behavior of masonry walls [3-7], strongly increase the safety level of masonry constructions under dynamic actions. A masonry wall can be regarded as a rigid block, if its behavior is monolithic, that is the mortar joints are in good condition and the masonry texture is sufficiently regular. A method, alternative to the kinematic analysis [810], that can be used for assessing the dynamic response of rigid blocks is a non-linear dynamic analysis, also called "rocking analysis", that is based on the pioneering Housner's work [11]. Due to the strong nonlinearities involved in the problem, given by the dynamics of the rigid block and by the boundary conditions (tie rods and transverse walls), the equation of motion of the rocking block must be solved step by step if the input action is a real seismic record. The motion of the block depends on its size and slenderness in a non-unique way. When dealing with masonry façades, one-sided motion should be considered in place of the two-sided motion, namely the effect of the transverse walls connected to the façade has to be taken into account. Giresini and Sassu [12] simulated the transverse walls as spring beds with a stiffness function of the masonry elastic modulus, thickness and effective depth of the sidewalls. In addition to the motion type, the response of rocking blocks is strongly dependent on the input action: even input actions with similar Intensity Measures (IM) values (e.g. PGA, PGV, Housner Intensity, etc.) may produce completely different results. This is a crucial aspect when one needs to analyse as realistic as possible.

For this reason, the necessity of introducing stochastic approaches in order to evaluate the response in probabilistic terms arises. Some efforts in this direction were recently made by Dimitrakopoulos and Paraskeva [13], where PGA and Peak Total Roof Velocity (PTRV) were identified as relevant IM for rocking parapets and rooftop chimneys under near-fault seismic actions. The same work also highlighted the superiority of bivariate fragility curves over univariate fragility curves.

In this paper, 4 cases of church masonry façades are analyzed, discussing the influence of size, slenderness and presence of tie-rods in the response. The analysis results are reported in terms of fragility curves, making considerations on the beneficial effects introduced by the tierods and presenting an efficient manner to display results with specific graphs. Section 2 introduces the deterministic equation of motion and the stochastic approach adopted. Section 3 describes the influence of slenderness and size on the response, considering in the OOP modes one-sided motion. Section 4 presents the outcomes of the same façades retrofitted by anti-seismic devices such as steel tie rods, commenting the range of IMs in which the retrofitting techniques have the best effects. Finally, section 5 discusses the comparisons between univariate and bivariate fragility curves.

\section{STOCHASTIC APPROACH FOR THE SEISMIC VULNERABILITY ASSESSMENT OF ROCKING FAÇADES}

\subsection{Assumptions in the deterministic analysis}

The stochastic approach can be defined once the corresponding procedure to perform the deterministic analysis is established. Assuming the mechanical and input parameters involved in the 
nonlinear dynamic analysis as deterministic, the motion of the generic prismatic block in unilateral condition can be obtained by solving the following equation of motion:

$$
\begin{gathered}
I_{0} \ddot{\vartheta}+\operatorname{sgn}(\vartheta) m g R \sin A_{\vartheta}+\operatorname{sgn}(\vartheta) K \beta^{2} R^{2} \cos A_{r, \vartheta}\left[\sin \alpha_{r}-\sin A_{r, \vartheta}\right] \\
+\operatorname{sgn}(\vartheta) K_{c}^{\prime} \bar{h}\left(A+\frac{B \bar{h}}{2}+\frac{C \bar{h}^{2}}{3}\right)-m \ddot{u}_{g} R \cos A_{\vartheta}=0
\end{gathered}
$$

where $A_{\vartheta}=\alpha-\operatorname{sgn}(\vartheta) \vartheta$ and $A_{r, \vartheta}=\alpha_{r}-\operatorname{sgn}(\vartheta) \vartheta . \alpha$ is the angle that defines the slenderness of the block (Figure 1a); $\alpha_{r}$ is the angle formed by the line along the block height and the restraint and $R_{r}$ is the single spring radius vector, whereas $\beta$ defines the single spring position $R_{r}=\beta R$ (Figure $1 \mathrm{~b}$ ). $\bar{h}$ is the effective depth along which the spring bed is effective. $I_{0}$ is the polar inertia moment with respect to the oscillation line O'-O (Figure 1a), $I_{0}=$ $\frac{4}{3} \mathrm{~m}\left(\mathrm{~h}^{2}+\mathrm{s}^{2}\right)=\frac{4}{3} \mathrm{mR}^{2}$, for perpendicular blocks.

However, inertia moments have to be calculated from the real geometry of the actual masonry façade. In this paper, the equivalent perpendicular block is obtained by keeping the same mass and inertia moment of the actual masonry façade. In this way, it is more effective to compare the equivalent radius vectors and slenderness ratios. As for the spring bed stiffness $K_{c}^{\prime}$, the terms multiplied by it are [12]:

$$
\begin{gathered}
A=\operatorname{sgn}(\vartheta) s^{2} \sin \vartheta \cos \vartheta(1-\cos \vartheta) \\
B=s\left(\sin ^{2} \vartheta \cos \vartheta-\cos ^{3} \vartheta+\cos ^{2} \vartheta\right) \\
C=\operatorname{sgn}(\vartheta) \sin \vartheta \cos ^{2} \vartheta
\end{gathered}
$$

The one-sided motion is considered, thus only the compression spring bed of stiffness $K_{c}^{\prime}$ is taken into account (inward rotation). Only in the retrofitted configuration, with steel tie -rods, the spring stiffness $K$ is different from zero. This occurs in the outward rotation, since the beneficial stabilizing effect given by the tie-rods is evident in the out-of-plane mode. The values of $K_{c}^{\prime}$ and $K$ are calculated with the expressions reported in [12].

As for the input excitation, $\ddot{u}_{g}$ is the acceleration time-history (in gravity acceleration units), which can be artificially generated or recorded. This work considers only natural seismic records as input for the activation of rocking motion.

During each impact, that is when $\vartheta=0$, the velocity after impact $\dot{\vartheta}^{+}$is assumed as the velocity before impact $\dot{\vartheta}^{-}$reduced by a coefficient of restitution $e$ :

$$
\dot{\vartheta}^{+}=\mathrm{e} \dot{\vartheta}^{-}
$$

The coefficient of restitution defined by Housner [14] for a rectangular block is a function of the slenderness ratio $\alpha$ :

$$
e_{\text {an }}=1-\frac{3}{2} \sin ^{2} \alpha
$$

For real masonry walls, the coefficient of restitution is usually lower than the analytical one $[15,16]$. However, in this paper the analytical value is considered for the sake of safety. Indeed, since it corresponds to a lower energy dissipation, the use of the analytical value usually causes greater rotation amplitudes. 


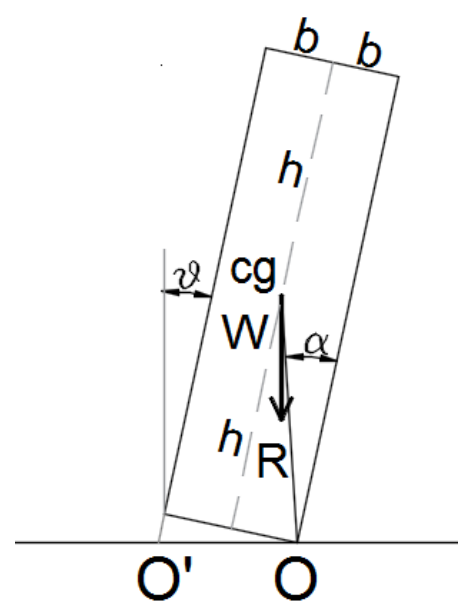

(a)

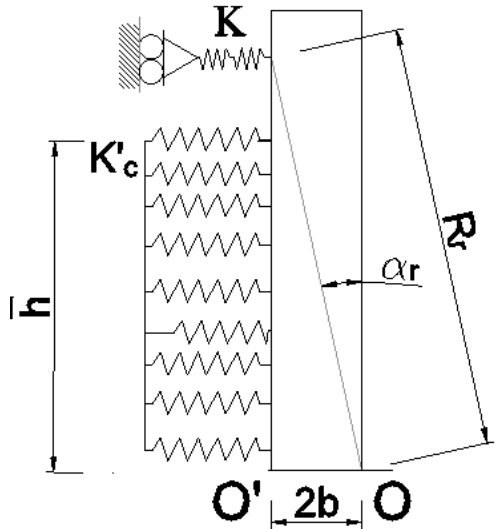

(b)

Figure 1: The rocking rigid block simulating an out-of-plane masonry wall in the Housner's model [17] (a) and the restrained rocking block with spring bed stiffness $K_{c}^{\prime}$ and spring stiffness $K(b)$.

\subsection{Probabilistic analysis and optimal Intensity Measures}

The probabilistic analysis is performed considering the one-sided motion of the cases of study (section 2.3) under over seventy earthquakes, whose details are reported in [18]. Univariate and bivariate fragility curves are obtained with the calculation of a conditional probability of failure, selecting as Engineering Demand Parameter EDP the maximum normalized rotation $\vartheta / \alpha$ and $12 \mathrm{IMs}$, which are related to the seismic inputs. The conditional probability is defined as the probability that EDP overcomes a capacity limit $C$, given a specific IM; such a probability is a standard cumulative distribution function [19].

As for the capacity limit, four limit states (from LS0 to LS3) are defined for the one-sided motion without horizontal restraints, and one for the state of tie-rod yielding LSY [18] (

Table 1). Naturally, the value of EDP only depends on the slenderness ratio $\alpha$ for LS0, LS1, LS2 and LS3, whereas for LSY it depends on the geometrical and mechanical features of the tie rod (elastic modulus, cross section and length).

Limit State LS $\quad E D P=\left(\vartheta_{\max } / \alpha\right)_{\text {lim }} \quad$ Definition

\begin{tabular}{ccc}
\hline$L S 0$ & 0.0 & rocking initiation \\
\hline$L S Y$ & Depending on geometry & tie-yielding \\
\hline$L S 1$ & 0.1 & limited rocking \\
\hline$L S 2$ & 0.4 & moderate rocking \\
\hline$L S 3$ & 1.5 & Near collapse
\end{tabular}

Table 1: Limit states assumed in the stochastic rocking analysis. 
Following the Padgett et al's procedure [19], the conditional probability is function of the logarithmic standard deviation $\beta_{D \mid I M}$, also called dispersion, of the demand conditioned on the IM level. Only demand uncertainties are taken into account in this work.

The relationship between the median structural demand (that is $E D P$ ) $S_{D}$ and IM is for univariate (conventional) fragility curves:

$$
S_{D}=a I M^{b}
$$

Bivariate fragility curves contain more information, due to the fact that they correlate the results with a pair of IMs. For bivariate fragility curves, the structural demand is expressed as:

$$
S_{D}=a I M_{1}^{b_{1}} \cdot I M_{2}^{b_{2}}
$$

The optimal IMs are those responding to the efficiency, proficiency, practicality, sufficiency and hazard computability criteria [19]. The first three criteria are considered in this article: as for the first one, the lower the dispersion coefficient $\beta_{D \mid I M}$, the more efficient the corresponding IM. Practicality is measured by the regression parameter $b$, which appears in Equations (5)-(6)): the greater this parameter, the better the correlation with the corresponding IM. Finally, the proficiency is measured through the parameter $\zeta=\beta_{D \mid I M} / b$, which therefore considers both efficiency and practicality. Pearson's and Spearman's coefficients can be recalled to select the best IMs. Indeed, the higher the correlation coefficient, the minor the dispersion and therefore the relevance of the IM.

\subsection{Cases of study}

The reference cases of this study are six masonry walls, among which three real church façades of different slenderness and size [18] and three additional reference façades of smaller dimensions ( $\mathrm{smCH}, \mathrm{smCHb}, \mathrm{smCHc}$,

Table 2). The churches were severely struck by the 2016-2017 Central Italy earthquakes exhibiting typical collapse mechanisms for masonry structures, among which the incipient OOP of the main façades. The three façades are not perfectly rectangular, but the walls have been idealized as prisms with equivalence criteria regarding the centers of mass and the inertia moments.

\begin{tabular}{ccccccccc}
$\begin{array}{c}\text { Rocking fa- } \\
\text { çade }\end{array}$ & $\begin{array}{c}\text { Equiv. height } \\
{[\mathrm{m}]}\end{array}$ & $\begin{array}{c}\text { Thickness } \\
{[\mathrm{m}]}\end{array}$ & $\begin{array}{c}\text { Width } \\
{[\mathrm{m}]}\end{array}$ & $\begin{array}{c}\text { Specific } \\
\text { weight } \\
{\left[\mathrm{kN} / \mathrm{m}^{3}\right]}\end{array}$ & $\begin{array}{c}\mathrm{R} \\
{[\mathrm{m}]}\end{array}$ & $\begin{array}{c}\alpha \\
{[\mathrm{rad}]}\end{array}$ & $\begin{array}{c}\mathrm{I}_{0} \\
{\left[\mathrm{~kg} \mathrm{~m} \mathrm{~m}^{2}\right]}\end{array}$ & $\begin{array}{c}\mathrm{K}_{\mathrm{c}}^{\prime} \\
{\left[\mathrm{N} / \mathrm{m}^{2}\right]}\end{array}$ \\
\hline $\mathrm{LSCH}$ & 8.85 & 0.65 & 9.3 & 21 & 4.44 & 0.07 & $\begin{array}{c}2.82 \mathrm{E}+ \\
06\end{array}$ & $\begin{array}{c}8.08 \mathrm{E}+ \\
08\end{array}$ \\
\hline SMVCH & 21.3 & 1.30 & 15.5 & 18 & 10.67 & 0.06 & $\begin{array}{c}1.27 \mathrm{E}+ \\
08\end{array}$ & $\begin{array}{c}1.02 \mathrm{E}+ \\
09\end{array}$ \\
\hline SFILCH & 7.64 & 0.80 & 12.0 & 18 & 3.84 & 0.10 & $\begin{array}{c}2.36 \mathrm{E}+ \\
06\end{array}$ & $\begin{array}{c}7.50 \mathrm{E}+ \\
08\end{array}$ \\
\hline $\mathrm{smCH}$ & 6.00 & 0.60 & 1.0 & 21 & 3.02 & 0.10 & $\begin{array}{c}9.80 \mathrm{E}+ \\
04\end{array}$ & $\begin{array}{c}8.08 \mathrm{E}+ \\
08\end{array}$ \\
\hline $\mathrm{smCHb}$ & 6.50 & 0.65 & 5.0 & 18 & 3.27 & 0.10 & $\begin{array}{c}4.00 \mathrm{E}+ \\
05\end{array}$ & $\begin{array}{c}8.08 \mathrm{E}+ \\
08\end{array}$ \\
\hline $\mathrm{smCHc}$ & 6.50 & 0.65 & 9.3 & 21 & 3.27 & 0.10 & $\begin{array}{c}1.20 \mathrm{E}+ \\
06\end{array}$ & $\begin{array}{c}8.08 \mathrm{E}+ \\
08\end{array}$ \\
\hline
\end{tabular}

Table 2: Geometrical and mechanical features of the façades. 
LSCH and SMVCH have similar slenderness but the latter has the highest inertia moment. Among the three real cases, SFILCH has the smallest radius vector and inertia moment, but the highest slenderness equal to 0.10 . The additional cases are selected by varying the specific weight, the equivalent height and thickness, whereas the slenderness ratio (ratio thickness/height) is the same and equal to that of SFILCH, i.e. 0.10.

As for the steel tie-rods, those of the church façades are designed according to what discussed in [18], whereas for the additional reference cases smaller values of stiffness are selected, in order to assess the efficiency of lower cross sections in the restraint effect during the one-sided rocking motion (Table 3). However, it is possible to observe that, for traditional tie-rods, the values of stiffness, is about $2 \mathrm{E} 7 \mathrm{~N} / \mathrm{m}$ [20] if a pair of 16-20 mm diameter steel tie-rods are used. Only for one case $(\mathrm{smCHb})$ a stiffness of one order of magnitude $(1 \mathrm{E} 6 \mathrm{~N} / \mathrm{m})$ smaller is assumed to state the effectiveness of such a device in the one-sided rocking motion.

\begin{tabular}{|c|c|c|c|c|c|}
\hline Rocking façade & $\begin{array}{c}\text { n. of tie-rods } \\
{[-]}\end{array}$ & $\begin{array}{c}\text { Tie diameter } \\
{[\mathrm{mm}]}\end{array}$ & $\begin{array}{c}\text { Tie length } \\
{[\mathrm{m}]}\end{array}$ & $\begin{array}{c}\text { Tie-rod arm from the ground } \\
{[\mathrm{m}]}\end{array}$ & $\begin{array}{c}K \\
{[\mathrm{~N} / \mathrm{m}]}\end{array}$ \\
\hline LSCH & 2 & 20 & 7 & 7.0 & $1.88 \mathrm{E}+07$ \\
\hline SMVCH & 2 & 20 & 6 & 15.0 & $2.20 \mathrm{E}+07$ \\
\hline SFILCH & 2 & 16 & 3 & 3.0 & $2.56 \mathrm{E}+07$ \\
\hline smCH & 2 & 20 & 7 & 6.5 & $1.88 \mathrm{E}+07$ \\
\hline smCHb & 1 & 14 & 3 & 6.5 & $1.00 \mathrm{E}+06$ \\
\hline smCHc & 2 & 20 & 7 & 6.5 & $1.88 \mathrm{E}+07$ \\
\hline
\end{tabular}

Table 3: Equivalent stiffness $K$ of the tie-rods assumed in the retrofitted configuration.

\section{INFLUENCE OF SLENDERNESS AND SIZE ON THE CONDITIONAL PROBABILITY}

The influence of the geometric dimensions, that is of slenderness and wall size, on the conditional probability can be discussed by comparing the univariate fragility curves (FCs) of the optimal IMs, according to the criteria discussed in section 2.2. Sometimes, it is not straightforward to compare FCs of different cases, since the optimal IMs can be diverse depending on the analysis.

As for the comparison of façades of the same slenderness, the first optimal IMs is the Peak Ground Velocity PGV. Indeed, the Pearson's and Spearman's coefficients for smCH are respectively 0.75 (the second greater after PGA) and 0.86 (the greatest).

The same coefficients for SFILCH, which is selected as reference case having the same slenderness of 0.10 , are respectively 0.79 and 0.91 (both the greatest ones).

It is clear from Figure 2a that the small façade portion $\mathrm{smCH}$, with slenderness ratio of 0.10 and inertia moment one order of magnitude lower than SFILCH, has higher probability of attainment of the three limit states (Table 1).

This confirms the fact that the greater the inertia moment, the greater the stability [21]. Indeed, the rotational inertia increases the seismic resistance of the rocking block with the square of the block size, whereas the seismic demand linearly increases with the block size, being expressed by the overturning moment. The same occurs if the second optimal IM for the cases under consideration, that is PGA, is considered (Figure 2b), although the differences of conditional probability are slightly lower. 


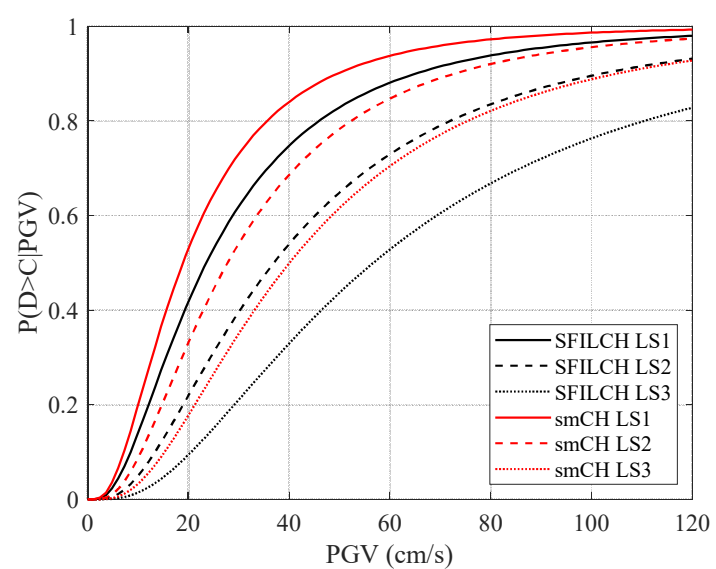

(a)

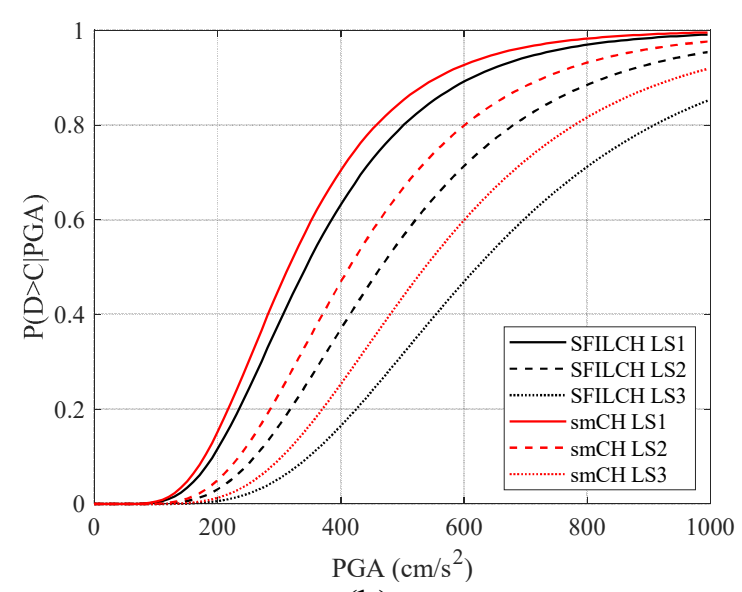

(b)

Figure 2: Comparison of façades of same slenderness $(0.10)$ and different inertia moments: IM=PGV (a) and $\mathrm{IM}=\mathrm{PGA}(\mathrm{b})$.

That occurs also if one compares LSCH and SMVCH (green and blue curves in Figure 5), that have about the same slenderness, but SMVCH has greater inertia moment and size. Nevertheless, in this case the differences in the conditional probability values are not so much evident for both IMs, especially for the limited rocking state LS1.

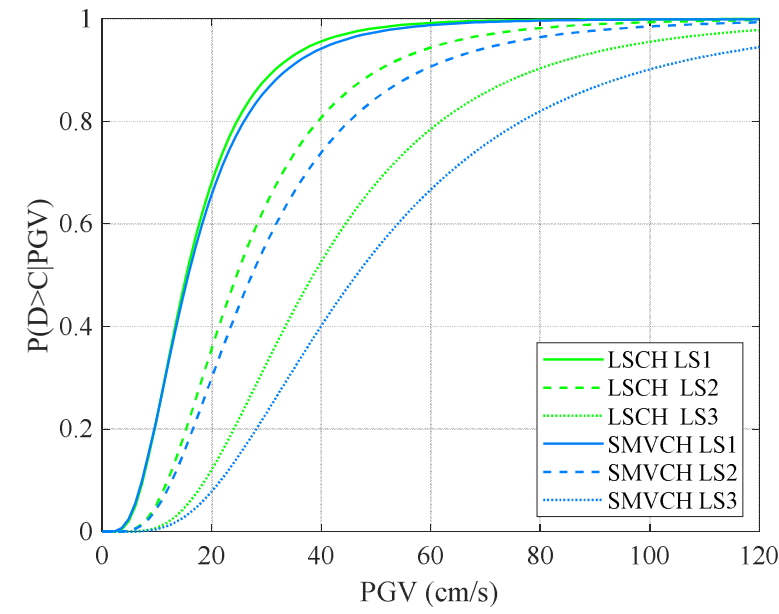

(a)

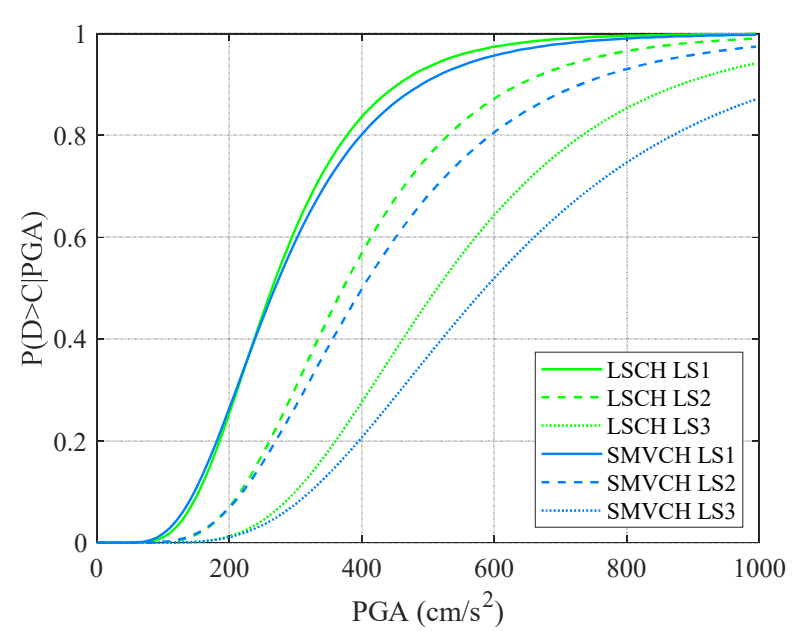

(b)

Figure 3: Comparison of façades of same slenderness (about 0.06) and different inertia moments: IM=PGV (a) and $\mathrm{IM}=\mathrm{PGA}(\mathrm{b})$. 


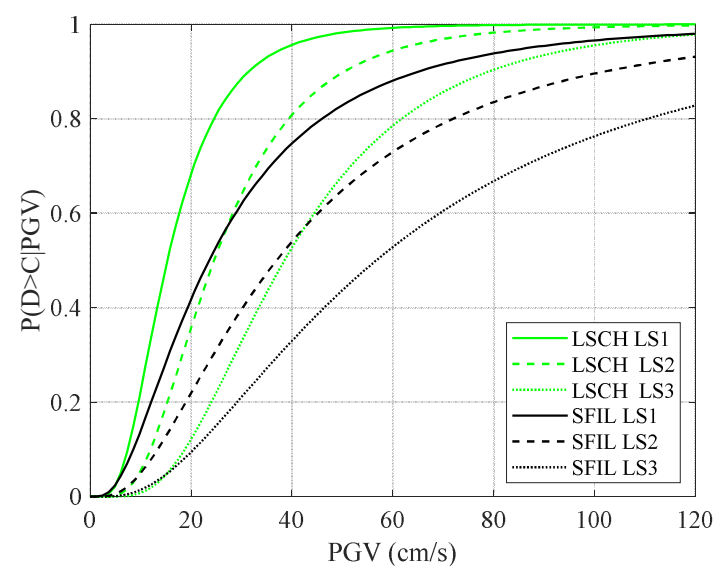

(a)

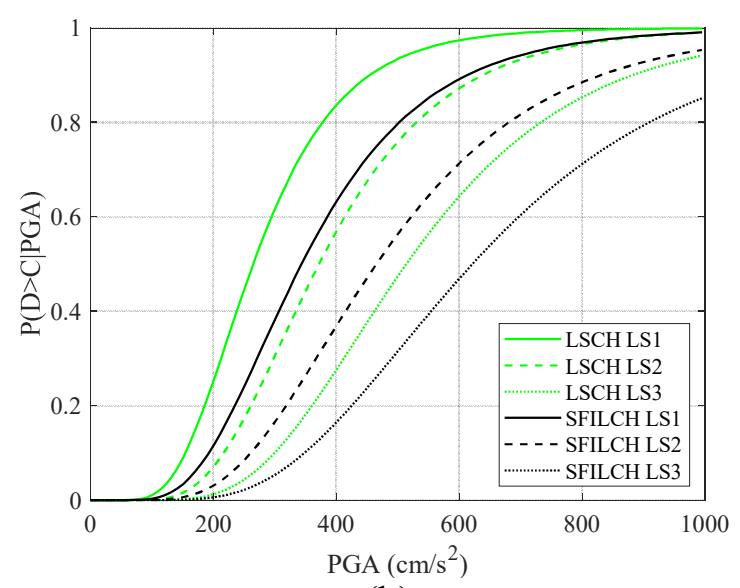

(b)

Figure 4: Comparison of façades of similar rotational inertia (about $2.5 \mathrm{E} 6 \mathrm{~kg} \mathrm{~m}^{2}$ ) and different slenderness ratios (LSCH $\alpha=0.07$, SFILCH $\alpha=0.10$ ): IM=PGV (a) and IM=PGA (b).

By considering façades of similar inertia, such as LSCH (green curve of Figure 4) and SFILCH (black curve), it is evident that the more slender case (LSCH) results in a more dangerous condition. Looking at the earthquake intensities, it is possible to observe that the stockier façade has a probability of overcoming the first limit state of limited rocking by $50 \%$ for values of PGV $>20 \mathrm{~cm} / \mathrm{s}$, that is for medium intensity earthquakes. Similarly, this $50 \%$ probability occurs for $P G A>0.3 \mathrm{~g}$.

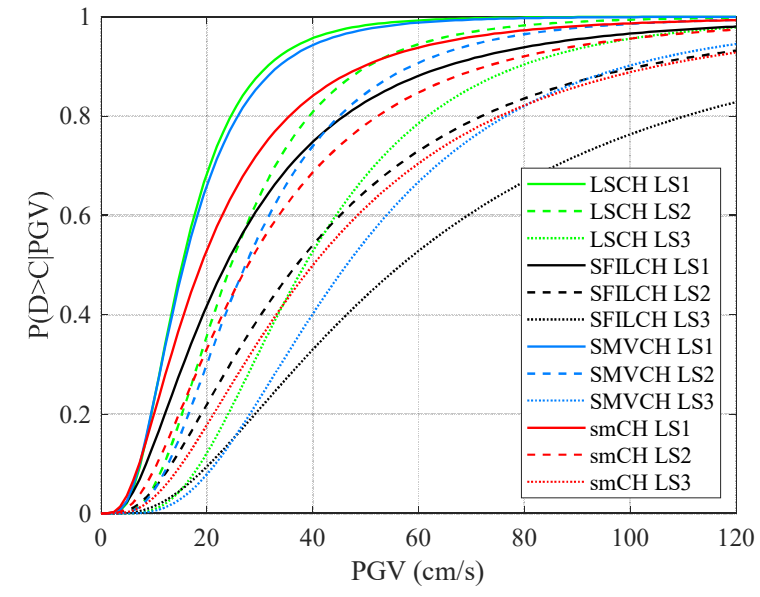

(a)

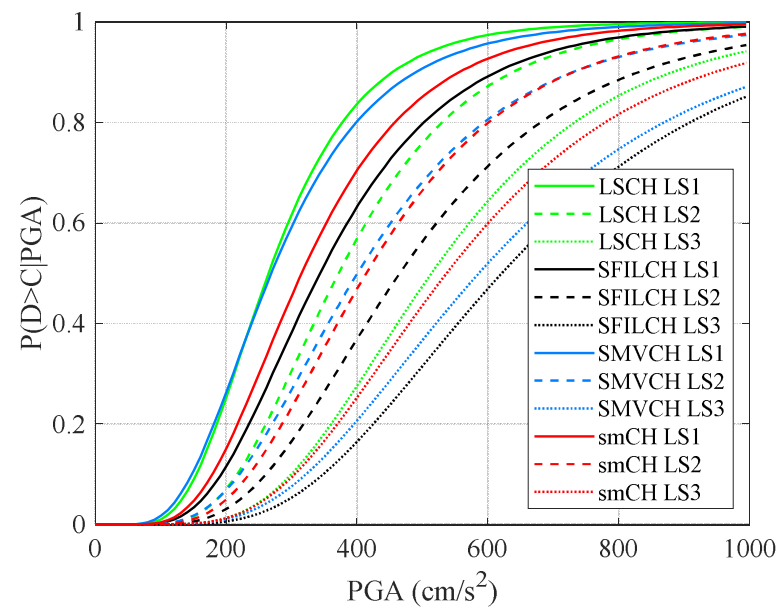

(b)

Figure 5: Univariate fragility curves for the four limit states in the condition of one-sided (1S) motion of all the façades with $\mathrm{IM}=\mathrm{PGV}$ (a) and $\mathrm{IM}=\mathrm{PGA}(\mathrm{b})$.

All the results for PGV and PGA are shown in Figure 5 and Figure 6. It is possible to observe that the most severe case is the church façade $\mathrm{LSCH}$, with slenderness ratio of 0.07 and inertia moment and size average among all cases (Table 1).

In general, these graphs are useful to state the upper bound of IM that the rocking wall can sustain with a specific conditional probability. 


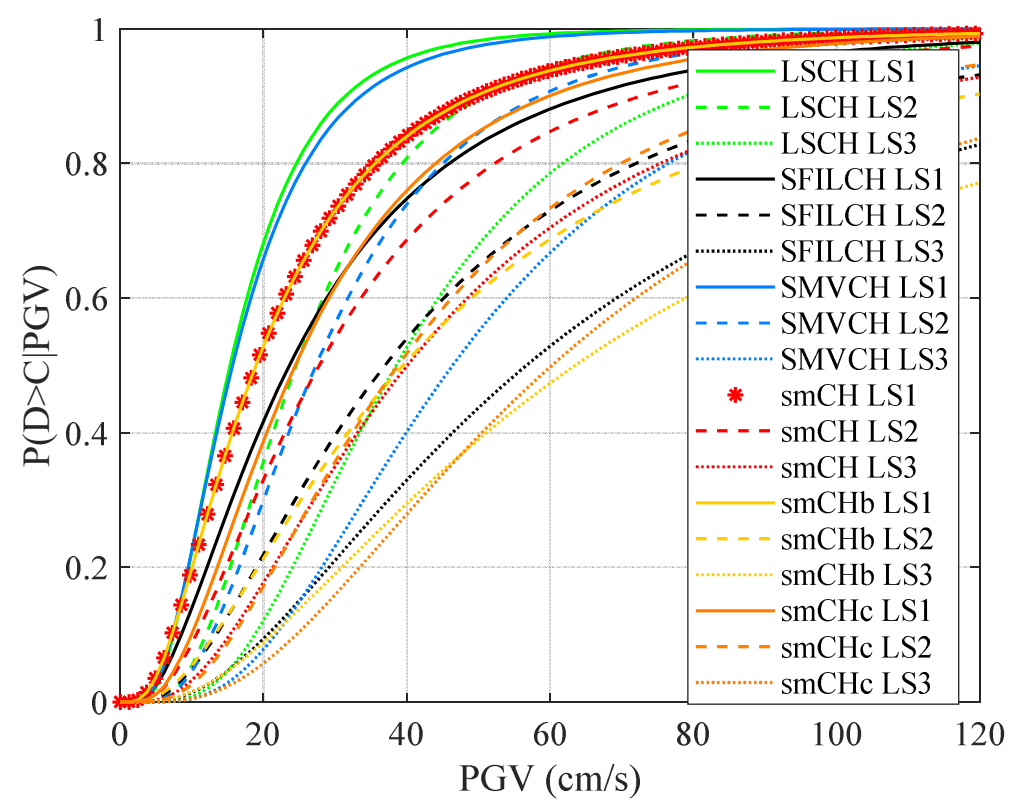

Figure 6: Univariate fragility curves for the four limit states in the condition of one-sided (1S) motion of all the façades with $\mathrm{IM}=\mathrm{PGV}$ (a) and IM=PGA (b).

\section{THE EFFECT OF ANTI-SEISMIC DEVICES ON THE CONDITIONAL PROBABILITY}

The examples of façades analyzed in the one-sided motion 1S, considering the transverse walls only in the inward rotation, are here discussed by restraining them with anti-seismic devices, such as steel tie-rods with stiffness $\mathrm{K}$ (Figure $1 \mathrm{~b}$ and Table 2). Some of the results were partially presented in [22] only for the real cases (LSCH, SMVCH and SFILCH). There are two ways of presenting the results in the as-built (without horizontal restraints in 1S) and in the retrofitted (with horizontal restraints still in $1 \mathrm{~S}$, that is $1 \mathrm{~S}+\mathrm{K}$ ): (i) compare the univariate FCs plotting the two configurations (e.g. Figure 7a) and (ii) plotting the difference of conditional probability (e.g. Figure 7b). This latter option is believed to be more effective and straightforward, since it reduces the number of output curves and it directly provide information about (a) the effectiveness of the designed tie-rods and (b) in which range of a specific IM the beneficial effect is maximized.

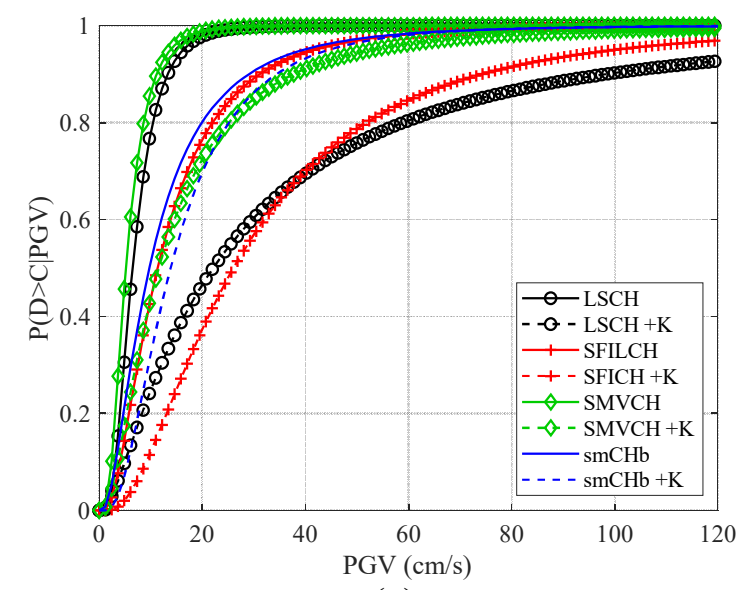

(a)

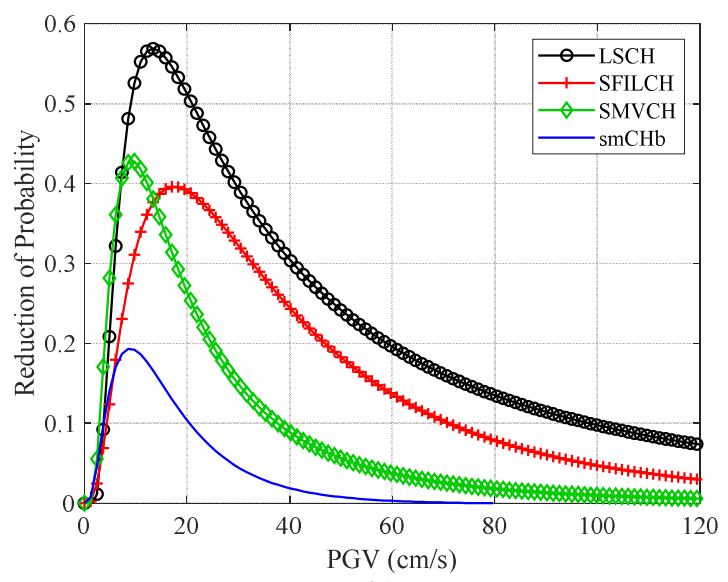

(b)

Figure 7: Univariate fragility curves for the yielding limit state with IM=PGV: conditional probability (a) and reduction of probability (b). 
It is possible to observe that the less beneficial effect is obtained by the case of slenderness ratio 0.10 and lower inertia moment (Figure $7 \mathrm{~b}$ ), among those plotted in the figure. This is likely due to the fact that the assumed stiffness for the steel tie-rod is not sufficient to properly restrain the wall. Indeed, this value of $1 \mathrm{E} 6 \mathrm{~N} / \mathrm{m}$ was chosen considering the reduced moment of inertia but it is out of the common range of stiffness corresponding to usually adopted tie-rods, as discussed in section 2.3 and in [23]. Nevertheless, the dynamic response in restrained configuration is in this case not sufficiently reduced by the anti-seismic device and more realistic values of stiffness are required (greater than $1 \mathrm{E} 7 \mathrm{~N} / \mathrm{m},[20]$ ). Indeed, considering the uncertainties due to the estimation of the mechanical parameters and to the calculations, at least an improvement of 30$40 \%$ should be recommended. Such an improvement is obtained for LSCH, SMVCH and SFIL for ranges of PGV between 10 and $20 \mathrm{~cm} / \mathrm{s}$ (Figure $7 \mathrm{~b}$ ) and $0.18-0.28 \mathrm{~g}$ (Figure 8 ) for the façade with greatest inertia moment and size (diamond green curves). The façade with the range where the improvement of seismic behavior is maximized is the LSCH, which is pretty typical as size and slenderness in the Italian architectural heritage of single-nave churches.

The maximum reduction of probability of overcoming LSY is about 55\% in both cases of IMs. The maximum improvement obtained in probabilistic terms is related to low-medium intensity earthquakes.

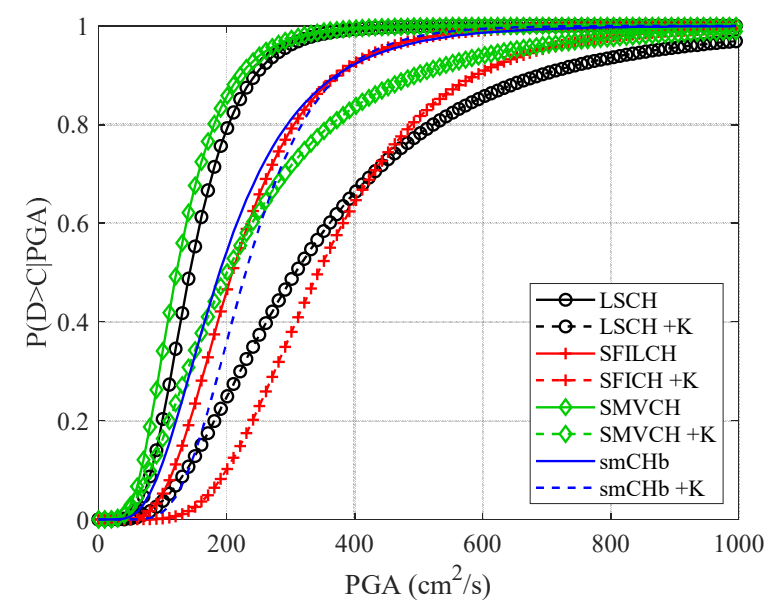

(a)

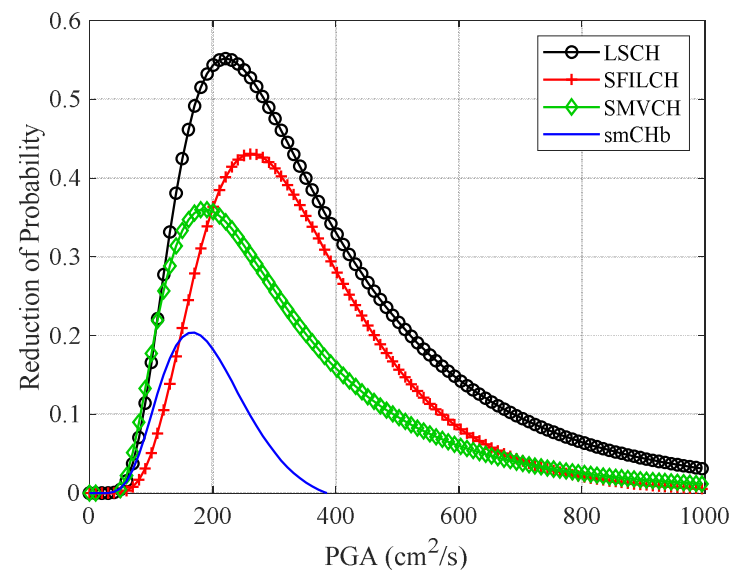

(b)

Figure 8: Univariate fragility curves for the yielding limit state with IM=PGA: conditional probability (a) and reduction of probability (b) from the one-sided motion to the restrained one-sided motion.

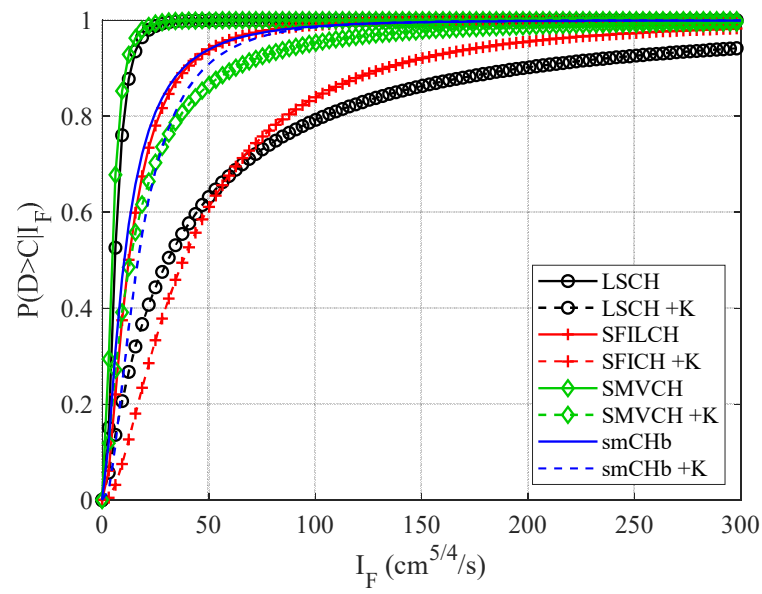

(a)

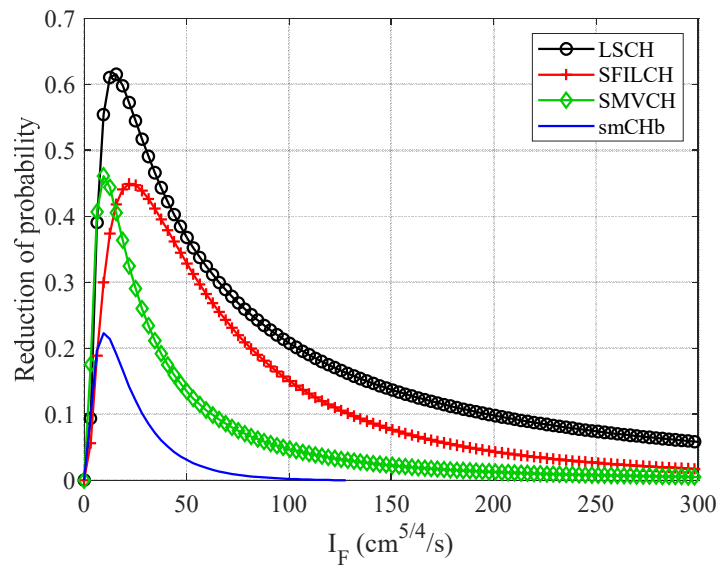

(b)

Figure 9: Univariate fragility curves for the yielding limit state with $\mathrm{IM}=\mathrm{I}_{\mathrm{F}}$ : conditional probability (a) and reduction of probability (b) from the one-sided motion to the restrained one-sided motion. 


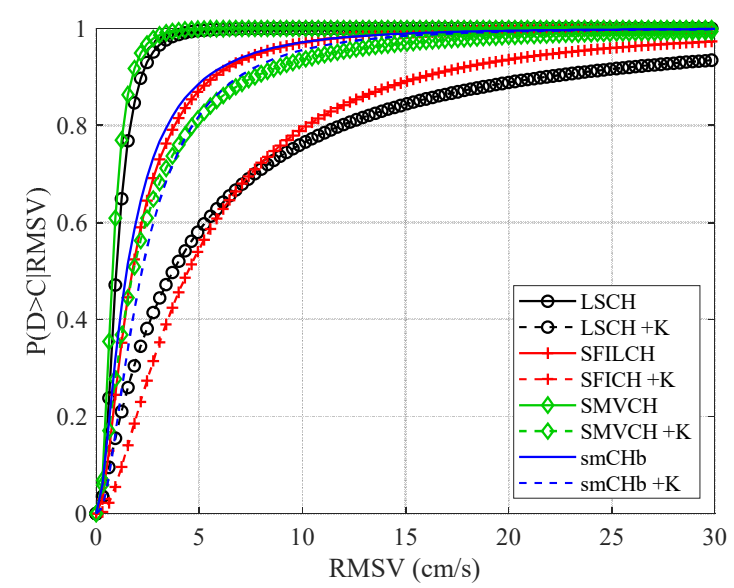

(a)

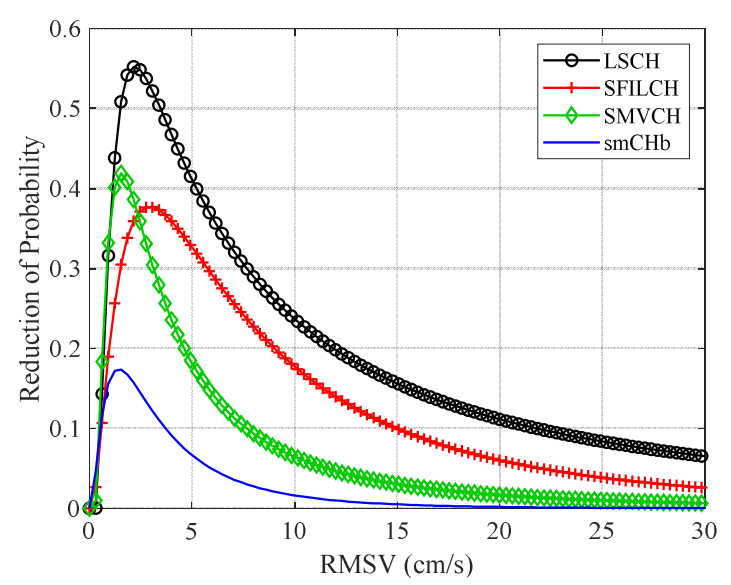

(b)

Figure 10: Univariate fragility curves for the yielding limit state with IM=RMSV: conditional probability (a) and reduction of probability (b) from the one-sided motion to the restrained one-sided motion.

\section{DISCUSSION ON UNIVARIATE AND BIVARIATE CURVES}

The selection of the couple of IMs for the bivariate FCs is not straightforward. A possible criterion of selection is that of coupling velocity based and acceleration based IMs, or velocity based and energy based IMs. Indeed, from the analysis of the Pearson's and Spearman's coefficients, generally these IMs are the most relevant.

A more logical criterion is that of identifying the pair of IMs corresponding to the minimum values of the dispersion coefficient or logarithmic standard deviation $\beta_{D \mid I M}$. As discussed for the univariate FCs, for different cases the minimum dispersion coefficients vary and therefore it is not possible to carry out a direct comparison.

For instance, for LSCH the minimum dispersion coefficient is the couple Arias Intensity and Root Mean Square of Displacement RMSD, whereas the same case in retrofitted configuration (that is with steel tie-rods) is the Arias Intensity and the Cumulative Absolute Velocity CAV. Another example is given by SMVCH, where the IMs pair with lower dispersion is the Arias Intensity and the Root Mean Square Velocity RMSV, whilst for the case with horizontal restraints is the Energy Density $I_{V}$ and the Fajfar Index $I_{F}$.

For some horizontal restraints, for instance if they have not enough stiffness to properly restrain the rocking wall (as seen also in the univariate case for $\mathrm{smCHb}$ in section 4 ) the conditional probability in the bivariate FC can be greater than the conditional probability without restraints (Figure 11a). This results in a negative difference of probability of attainment of the yielding limit state that indicates a null beneficial effect (or even negative) offered by the antiseismic device (Figure 11b). However, for low intensity earthquakes (say $\mathrm{PGV}<30 \mathrm{~cm} / \mathrm{s}$ ) the positive effect is visible with a maximum reduction of $20 \%$.

A much greater improvement is seen for LSCH (Figure 12) with maximum reduction of conditional probability of more than $80 \%$ for low-medium intensity earthquakes. 


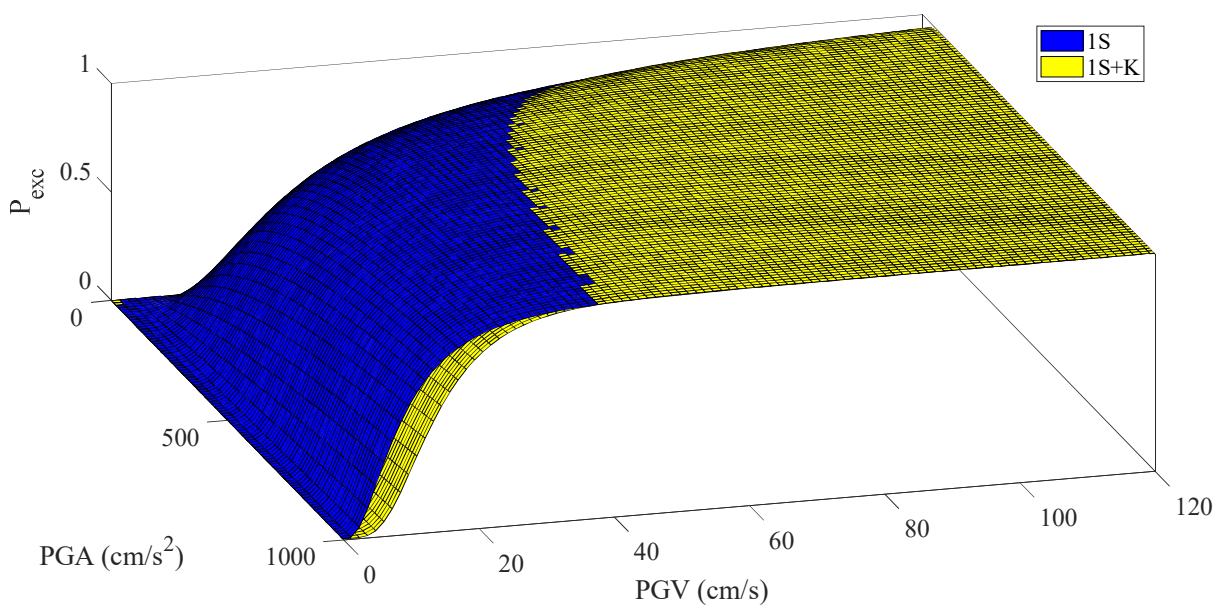

(a)

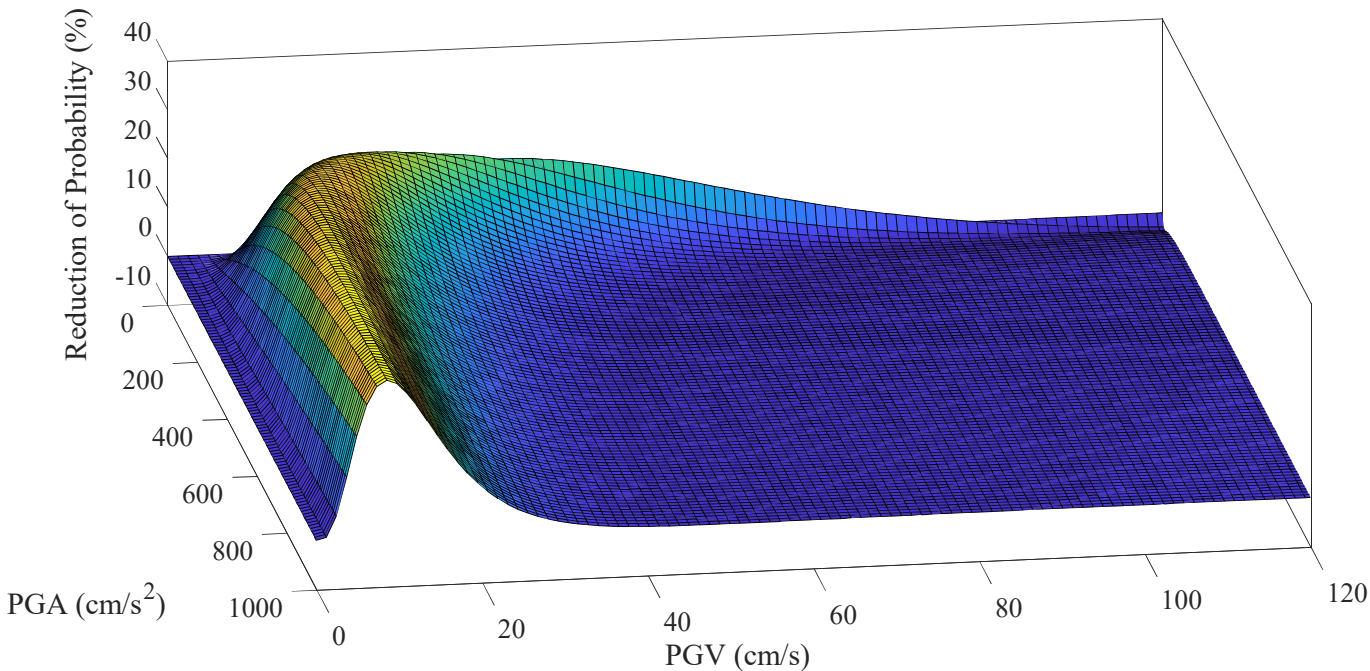

(b)

Figure 11: Bivariate fragility curves for the yielding limit state of smCHb: conditional probability (a) and reduction of probability (b) from the one-sided motion to the restrained one-sided motion.

To state the superiority of bivariate curves over univariate curves, some results are plotted by selecting bivariate FCs and fixing one of the two IMs and comparing the variable ones with the corresponding univariate FC.

An example is shown in Figure 13: it is clear that the univariate FC underestimates the probability of attainment of the moderate rocking limit state LS2 and in one case (a) is flatten and therefore improper. 


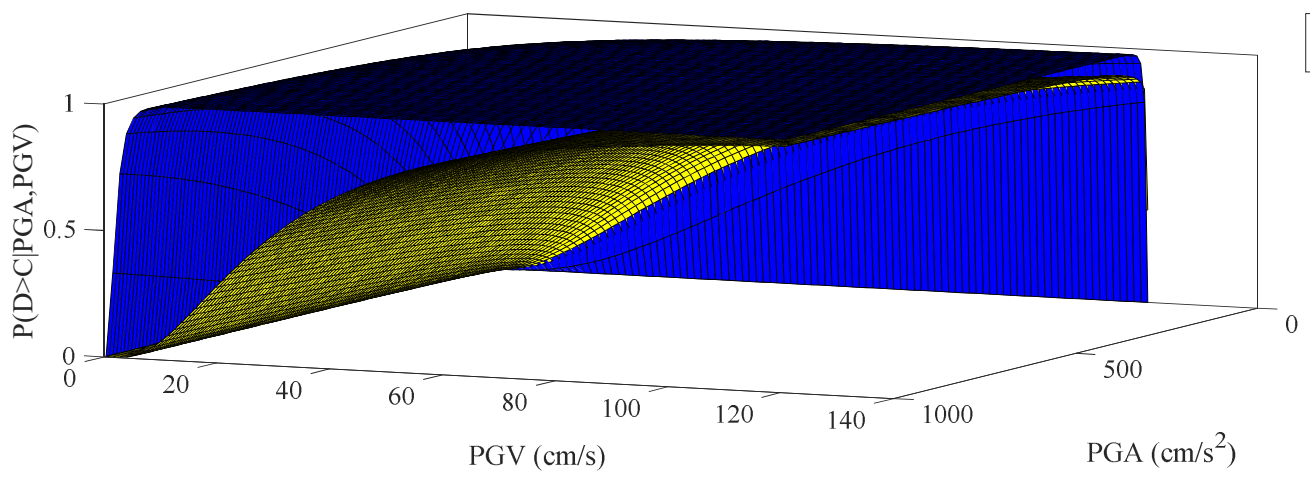

(a)

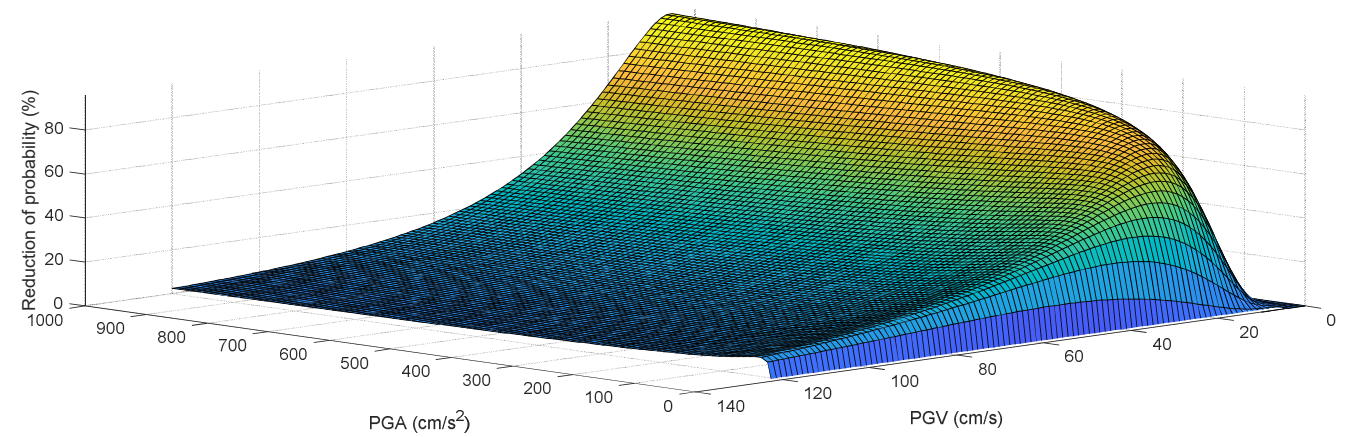

(b)

Figure 12: Bivariate fragility curves for the yielding limit state of LSCH: conditional probability (a) and reduction of probability (b) from the one-sided motion to the restrained one-sided motion.

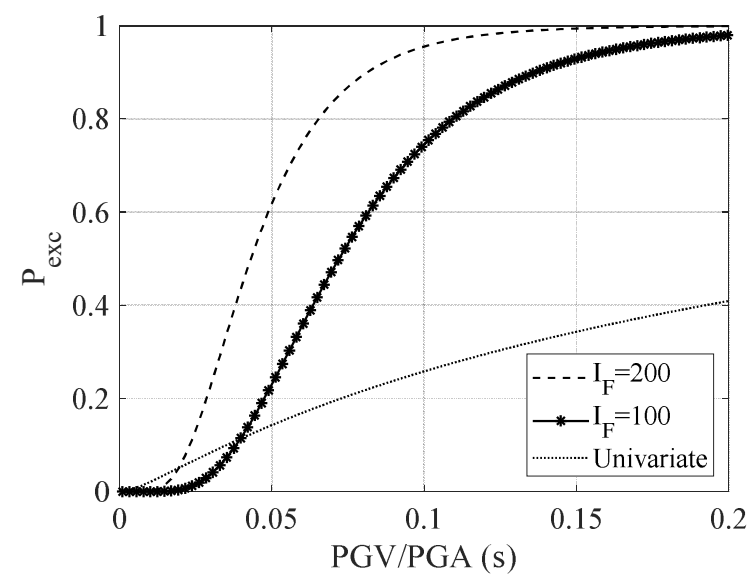

(a)

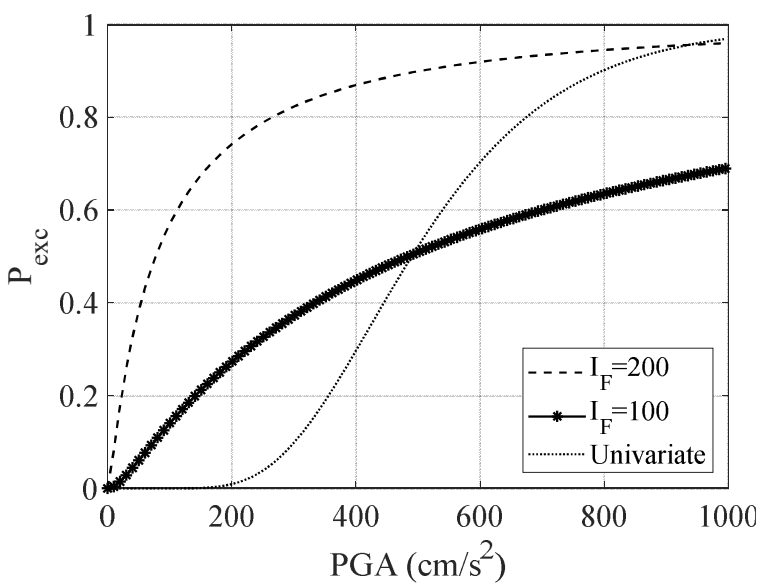

(b)

Figure 13: Bivariate and univariate fragility curves for moderate rocking (LS2) of smCHc: PGV/PGA for different IF values (a); PGA for different IF values (b). 


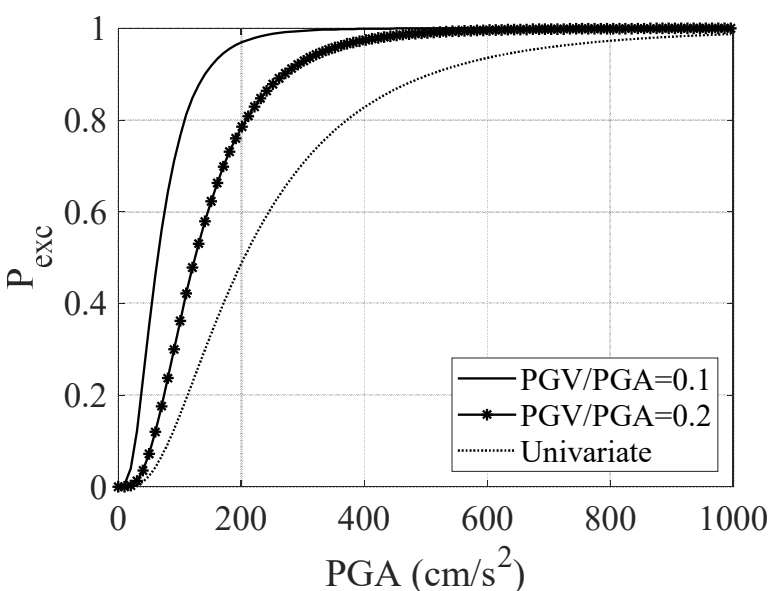

(a)

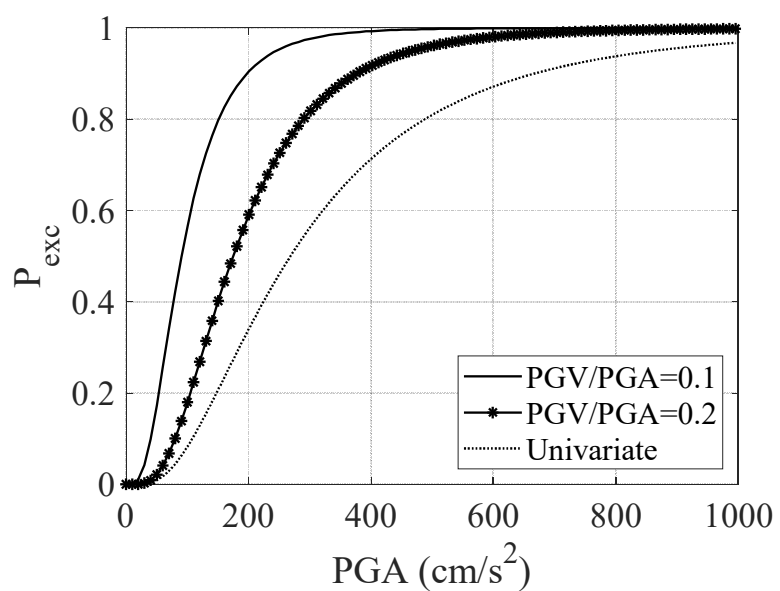

(b)

Figure 14: Bivariate and univariate fragility curves for SFILCH: LS1 (a); LS2 (b).

In other cases, although the univariate FC has a proper shape, it still underestimates the conditional probability (dotted lines in Figure 14). Such an underestimation reaches values of about $20 \%$ for medium intensity earthquakes, and therefore in some cases the univariate FCs give unacceptable results.

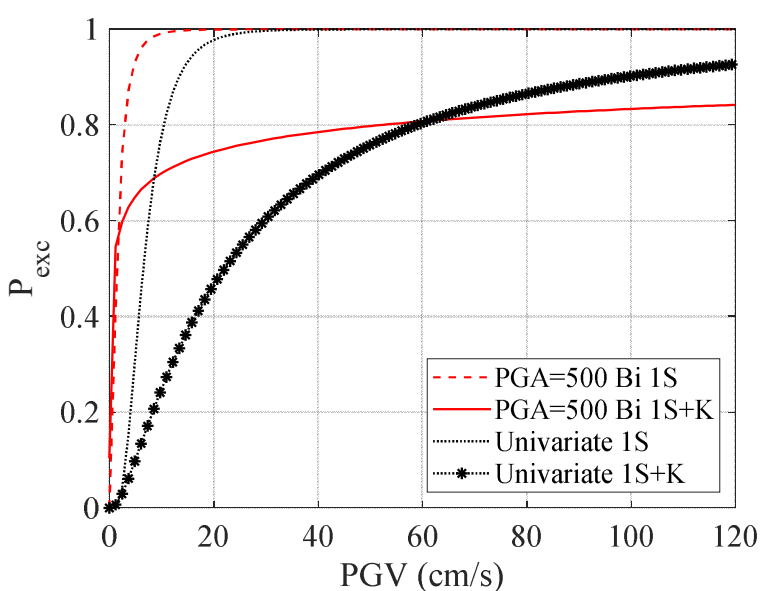

(a)

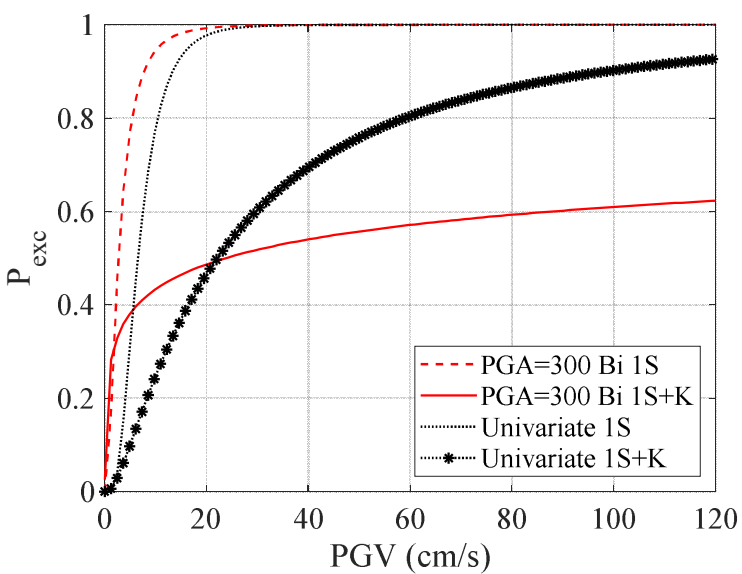

(c)

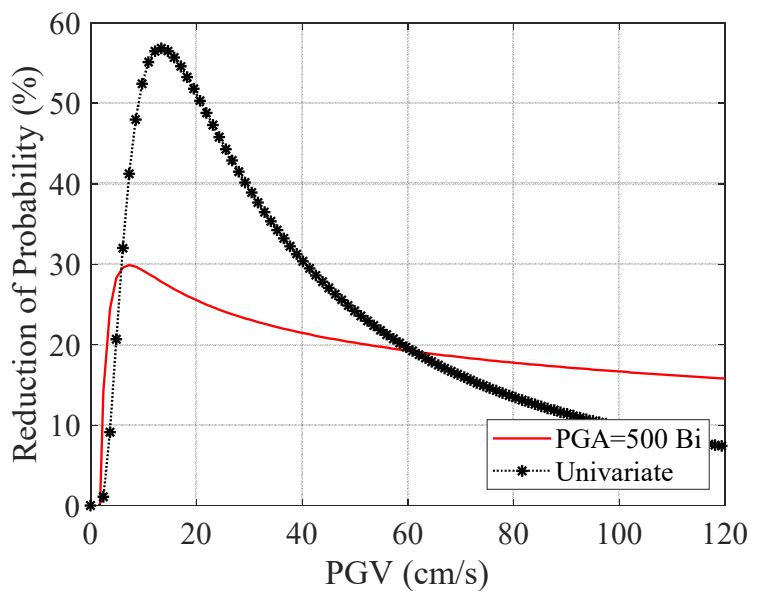

(b)

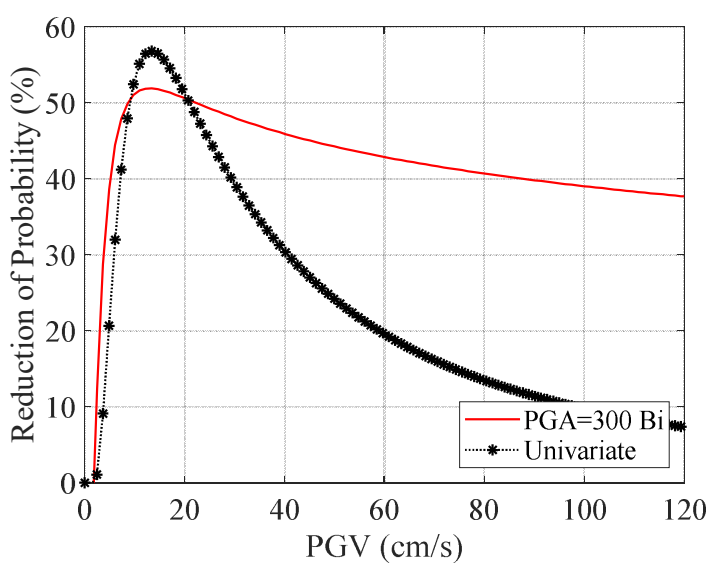

(d)

Figure 15: Bivariate and univariate fragility curves for LSCH, LSY: conditional probability and difference of probability for $\mathrm{PGA}=500 \mathrm{~cm} / \mathrm{s}^{2}(\mathrm{a}, \mathrm{b})$ and $\mathrm{PGA}=300 \mathrm{~cm} / \mathrm{s}^{2}(\mathrm{c}, \mathrm{d})$. 
A relevant comparison is believed to be that between the one-sided motion without horizontal restraints and that with horizontal restraints: the underestimation of the conditional probability in the retrofitted configuration would be quite risky in the seismic vulnerability assessment and therefore must be avoided. It is clearly visible from Figure 15 that the bivariate FCs are conservative since the associated probability is much higher than the univariate FCs in both one-sided and one-sided with tie-rods. The graph plotting the reduction of probability allows directly evaluating where the underestimation of the univariate FCs is more evident (for $5<\mathrm{PGV}<60 \mathrm{~cm} / \mathrm{s}$, Figure $15 \mathrm{a}, \mathrm{b})$. By contrast, for high intensity earthquakes (PGV $>60 \mathrm{~cm} / \mathrm{s}$ ), the univariate $\mathrm{FC}$ is more conservative than the bivariate one (Figure 15b). This effect is more stressed for low intensity values of PGA (Figure 15c,d): when one has high PGV value, the univariate FC can be more conservative. However, in general for real seismic records, very high values of PGV are associated to high values of PGA.

\section{CONCLUSIONS}

A stochastic approach was used in the one-sided rocking analysis of masonry façade with and without horizontal restraints to discuss the influence of geometrical and mechanical parameters. The conditional probability of attainment of five limit states, from the rocking onset to the collapse state, was calculated for different masonry façades. The optimal IMs identified are the Peak Ground Velocity PGV, Peak Ground Acceleration PGA and other velocity and energybased parameters as, e.g., the Fajfar Index and the Housner's intensity.

The influence of the geometric dimensions (slenderness and wall size) on the conditional probability was discussed by comparing the univariate fragility curves (FCs) of the optimal Intensity Measures (IMs). The comparison of results confirmed that the greater the inertia moment (with the same slenderness), the greater the stability also in stochastic terms. By considering façades of similar inertia, the more slender case is in a more dangerous condition for the attainment of all the limit states.

The univariate FCs graphs can be useful to state the upper bound of IM that the rocking wall can sustain with a specific conditional probability.

In addition to these graphs, when the beneficial effect of anti-seismic devices has to be quantified, specific curves of difference of conditional probability pre and post the retrofitting intervention were plotted. The maximum reduction of probability of overcoming LSY was for all cases about $55 \%$, by considering a pair of common steel-tie rods. The maximum improvement obtained in probabilistic terms is related to low-medium intensity earthquakes.

Univariate and bivariate curves were finally compared: the latter are conservative with respect to the univariate fragility curves, and therefore their use is recommended, especially whenever sophisticated considerations (e.g. for monumental façades) have to be performed. Indeed, the underestimation observed in the univariate FCs reaches values of about $20 \%$ for medium intensity earthquakes. In general, it could be useful to couple velocity based and acceleration based intensity measures, or velocity based and energy based intensity measures. For some horizontal restraints, for instance if they have not enough stiffness to properly restrain the rocking wall the difference of probability of attainment of the yielding limit state can be negative, indicating a null - or even detrimental - beneficial effect offered by the anti-seismic device.

\section{ACKNOWLEDGEMENTS}

The authors acknowledge the sponsorship of the Italian Civil Protection, through the RELUIS Project - Line: Masonry Structures. 


\section{REFERENCES}

1. Alecci V, De Stefano M. Building irregularity issues and architectural design in seismic areas. Frattura Ed Integrità Strutturale 2019; 13(57): 161-168.

2. Alecci V, De Stefano M, Galassi S, Lapi M, Orlando M. Evaluation of the American Approach for Detecting Plan Irregularity. Advances in Civil Engineering 2019.

3. De Falco A, Giresini L, Sassu M. Temporary preventive seismic reinforcements on historic churches: numerical modeling of San Frediano in Pisa. Applied Mechanics and Materials 2013; 352.

4. Giresini L. Energy-based method for identifying vulnerable macro-elements in historic masonry churches. Bulletin of Earthquake Engineering 2016; 14(3): 919-942. DOI: 10.1007/s10518-015-9854-7.

5. Giresini L, Sassu M, Butenweg C, Alecci V, De Stefano M. Vault macro-element with equivalent trusses in global seismic analyses. Earthquake and Structures 2017; 12(4): 409-423. DOI: 10.12989/eas.2017.12.4.409.

6. De Falco A, Giresini L, Sassu M. Structural analysis and consolidation strategy of the historic Mediceo Aqueduct in Pisa (Italy). Applied Mechanics and Materials 2013; 352: 1393-1396.

7. Andreini M, De Falco A, Giresini L, Sassu M. Collapse of the historic city walls of Pistoia (Italy): causes and possible interventions. Applied Mechanics and Materials 2013; 352: 1389-1392.

8. Casapulla C. La valutazione della sicurezza sismica delle chiese a scala territoriale, in: Il Patrimonio architettonico ecclesiastico di Napoli: Forme e spazi ritrovati, 2016, Publisher: Artstudio Paparo, pp.185-191.

9. Casapulla C, Maione A, Argiento LU, Speranza E. Corner failure in masonry buildings: An updated macro-modeling approach with frictional resistances. European Journal of Mechanics, A/Solids 2018; 70. DOI: 10.1016/j.euromechsol.2018.03.003.

10. Casapulla C, Maione A. Experimental and Analytical Investigation on the Corner Failure in Masonry Buildings: Interaction between Rocking-Sliding and Horizontal Flexure. International Journal of Architectural Heritage 2018. DOI: 10.1080/15583058.2018.1529206.

11. Casapulla C. On the resonance conditions of rigid rocking blocks. International Journal of Engineering and Technology 2015; 7(2): 760-771.

12. Giresini L, Sassu M. Horizontally restrained rocking blocks: evaluation of the role of boundary conditions with static and dynamic approaches. Bulletin of Earthquake Engineering 2017; 15(1): 385-410. DOI: 10.1007/s10518-016-9967-7.

13. Dimitrakopoulos EG, Paraskeva TS. Dimensionless fragility curves for rocking response to near-fault excitations. Earthquake Engineering \& Structural Dynamics 2015; 44(12): 2015-2033. DOI: 10.1002/eqe.2571.

14. Housner GW. The behavior of inverted pendulum structures during earthquakes. Bulletin of the Seismological Society of America 1963; 53(2): 403-417. DOI: 10.1017/CBO9781107415324.004. 
15. Giresini L, Sassu M, Sorrentino L. In situ free-vibration tests on unrestrained and restrained rocking masonry walls. Earthquake Engineering \& Structural Dynamics 2018; 47(15): 3006-3025. DOI: 10.1002/eqe.3119.

16. Sorrentino L, AlShawa O, Decanini LD. The relevance of energy damping in unreinforced masonry rocking mechanisms. Experimental and analytic investigations. Bulletin of Earthquake Engineering 2011; 9(5): 1-26.

17. Housner GW. The behavior of inverted pendulum structures during earthquakes. Bulletin of the Seismological Society of America 1963; 53(2): 403-417. DOI: 10.1017/CBO9781107415324.004.

18. Giresini L, Casapulla C, Denysiuk R, Matos J, Sassu M. Fragility curves for free and restrained rocking masonry façades in one-sided motion. Engineering Structures 2018; 164: 195-213. DOI: 10.1016/j.engstruct.2018.03.003.

19. Padgett JE, Nielson BG, DesRoches R. Selection of optimal intensity measures in probabilistic seismic demand models of highway bridge portfolios. Earthquake Engineering \& Structural Dynamics 2008; 37(5): 711-725. DOI: 10.1002/eqe.782.

20. Giresini L, Fragiacomo M, Lourenço PB. Comparison between rocking analysis and kinematic analysis for the dynamic out-of-plane behavior of masonry walls. Earthquake Engineering and Structural Dynamics 2015; 44(13): 2359-2376. DOI: 10.1002/eqe. 2592 .

21. Makris N. The role of the rotational inertia on the seismic resistance of free-standing rocking columns and articulated frames. Bull Seismol Soc Am 2014; 104: 2226.

22. Giresini L, Casapulla C, Denysiuk R, Matos J, Sassu M. Fragility curves for free and restrained rocking masonry façades in one-sided motion. Engineering Structures 2018; 164: 195-213. DOI: https://doi.org/10.1016/j.engstruct.2018.03.003.

23. Giresini L, Fragiacomo M, Lourenço PB. Comparison between rocking analysis and kinematic analysis for the dynamic out-of-plane behavior of masonry walls. Earthquake Engineering \& Structural Dynamics 2015; 44(13): 2359-2376. DOI: 10.1002/eqe.2592. 